\title{
First observation of the hyper superheavy hydrogen ${ }_{\Lambda}^{6} \mathrm{H}$
}

\author{
M. Agnello a,b,1 L. Benussi ${ }^{\mathrm{c}, 1}$, M. Bertani ${ }^{\mathrm{c}, 1}$, H.C. Bhang ${ }^{\mathrm{d}, 1}$, \\ G. Bonomi ${ }^{\mathrm{e}, \mathrm{f}, 1}$, E. Botta ${ }^{\mathrm{g}, \mathrm{b}, *, 1}$, M. Bregant ${ }^{\mathrm{h}, \mathrm{i}, 1}, \mathrm{~T}$. Bressani ${ }^{\mathrm{g}, \mathrm{b}, 1}$,
} S. Bufalino ${ }^{\mathrm{b}, 1}$, L. Busso ${ }^{\mathrm{g}, \mathrm{b}, 1}$, D. Calvo ${ }^{\mathrm{b}, 1}$, P. Camerini ${ }^{\mathrm{h}, \mathrm{i}, 1}$, B. Dalena ${ }^{\mathrm{j}, 1}$, F. De Mori ${ }^{\mathrm{g}, \mathrm{b}, 1}$, G. D’Erasmo ${ }^{\mathrm{k}, \mathrm{l}, 1}$, F.L. Fabbri ${ }^{\mathrm{c}, 1}$, A. Feliciello ${ }^{\mathrm{b}, 1}$, A. Filippi ${ }^{\mathrm{b}, 1}$, E.M. Fiore ${ }^{\mathrm{k}, 1,1}$, A. Fontana ${ }^{\mathrm{f}, 1}$, H. Fujioka ${ }^{\mathrm{m}, 1}$, P. Genova ${ }^{\mathrm{f}, 1}$, P. Gianotti ${ }^{\mathrm{c}, 1}$, N. Grion ${ }^{\mathrm{i}, 1}$, V. Lucherini ${ }^{\mathrm{c}, 1}, \mathrm{~S}$. Marcello ${ }^{\mathrm{g}, \mathrm{b}, 1}$, N. Mirfakhrai ${ }^{\mathrm{n}, 1}$, F. Moia ${ }^{\mathrm{e}, \mathrm{f}, 1}$, O. Morra ${ }^{\mathrm{o}, \mathrm{b}, 1}$, T. Nagae $^{\mathrm{m}, 1}$, H. Outa $^{\mathrm{p}, 1}$,

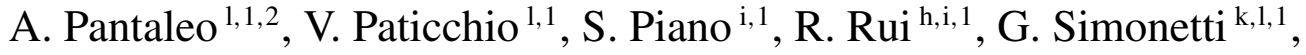
R. Wheadon ${ }^{\mathrm{b}, 1}$, A. Zenoni ${ }^{\mathrm{e}, \mathrm{f}, 1}, \mathrm{~A}$. Gal ${ }^{\mathrm{q}}$

a Dipartimento di Fisica, Politecnico di Torino, Corso Duca degli Abruzzi 24, Torino, Italy

b INFN Sezione di Torino, via P. Giuria 1, Torino, Italy

c Laboratori Nazionali di Frascati dell'INFN, via E. Fermi, 40, Frascati, Italy

d Department of Physics, Seoul National University, 151-742 Seoul, South Korea

e Dipartimento di Ingegneria Meccanica e Industriale, Università di Brecia, via Valotti 9, Brescia, Italy

${ }^{\mathrm{f}}$ INFN Sezione di Pavia, via Bassi 6, Pavia, Italy

g Dipartimento di Fisica, Università di Torino, via P. Giuria 1, Torino, Italy

${ }^{\mathrm{h}}$ Dipartimento di Fisica, Università di Trieste, via Valerio 2, Trieste, Italy

${ }^{\mathrm{i}}$ INFN Sezione di Trieste, via Valerio 2, Trieste, Italy

j CEA, Irfu/SACM, Gif-sur-Yvette, France

${ }^{\mathrm{k}}$ Dipartimento di Fisica, Università di Bari, via Amendola 173, Bari, Italy

${ }^{1}$ INFN Sezione di Bari, via Amendola 173, Bari, Italy

${ }^{m}$ Department of Physics, Kyoto University, Sakyo-ku, Kyoto, Japan

${ }^{\mathrm{n}}$ Department of Physics, Shahid Behesty University, 19834 Teheran, Iran

${ }^{\circ}$ INAF-IFSI, Sezione di Torino, Corso Fiume 4, Torino, Italy

p RIKEN, Wako, Saitama 351-0198, Japan

${ }^{\mathrm{q}}$ Racah Institute of Physics, The Hebrew University, Jerusalem 91904, Israel

Received 20 December 2011; received in revised form 23 February 2012; accepted 24 February 2012

Available online 2 March 2012

\footnotetext{
* Corresponding author at: INFN Sezione di Torino, via P. Giuria 1, Torino, Italy.

E-mail address: botta@to.infn.it (E. Botta).

1 FINUDA Collaboration.

2 Deceased.
} 


\begin{abstract}
Three candidate events of the neutron-rich hypernucleus ${ }_{\Lambda}^{6} \mathrm{H}$ were uniquely identified in the FINUDA experiment at DA $\Phi$ NE, Frascati, by observing $\pi^{+}$mesons from the $\left(K_{\text {stop }}^{-}, \pi^{+}\right)$production reaction on ${ }^{6} \mathrm{Li}$ targets, in coincidence with $\pi^{-}$mesons from ${ }_{\Lambda}^{6} \mathrm{H} \rightarrow{ }^{6} \mathrm{He}+\pi^{-}$weak decay. Details of the experiment and the analysis of its data are reported, leading to an estimate of $(2.9 \pm 2.0) \cdot 10^{-6} / K_{\text {stop }}^{-}$for the ${ }_{\Lambda}^{6} \mathrm{H}$ production rate times the two-body $\pi^{-}$weak decay branching ratio. The ${ }_{\Lambda}^{6} \mathrm{H}$ binding energy with respect to ${ }^{5} \mathrm{H}+\Lambda$ was determined jointly from production and decay to be $B_{\Lambda}=(4.0 \pm 1.1) \mathrm{MeV}$, assuming that ${ }^{5} \mathrm{H}$ is unbound with respect to ${ }^{3} \mathrm{H}+2 n$ by $1.7 \mathrm{MeV}$. The binding energy determined from production is higher, in each one of the three events, than that determined from decay, with a difference of $(0.98 \pm 0.74) \mathrm{MeV}$ here assigned to the $0_{\mathrm{g} . \mathrm{s} .}^{+} \rightarrow 1^{+}$excitation. The consequences of this assignment to $\Lambda$ hypernuclear dynamics are briefly discussed.
\end{abstract}

(C) 2012 Elsevier B.V. All rights reserved.

Keywords: Neutron-rich $\Lambda$-hypernuclei; Neutron drip line; Neutron halo phenomena

\title{
1. Introduction
}

The rôle of the $\Lambda$ hyperon in stabilizing nuclear cores was pointed out long ago by Dalitz and Levi Setti [1] as part of a discussion focusing on light hypernuclei with large neutron excess. This property is demonstrated by the observation of ${ }_{\Lambda}^{6} \mathrm{He},{ }_{\Lambda}^{7} \mathrm{Be},{ }_{\Lambda}^{8} \mathrm{He},{ }_{\Lambda}^{9} \mathrm{Be}$ and ${ }_{\Lambda}^{10} \mathrm{~B}$ hypernuclei in emulsion experiments [2]. No unstable-core hydrogen $\Lambda$ hypernuclei have been established so far, although the existence of the lightest possible one ${ }_{\Lambda}^{6} \mathrm{H}$ was predicted by Dalitz and Levi Setti [1] and subsequently reinforced in estimates by Majling [3]. The neutral-baryon excess in ${ }_{4}^{6} \mathrm{H}$, in particular, would be $(N+Y) / Z=5$, with $Y=1$ for a $\Lambda$ hyperon, larger than the maximal value in light nuclei, $N / Z=3$ for ${ }^{8} \mathrm{He}$ [4]. Neutron-rich light hypernuclei could thus go beyond the neutron drip line for ordinary nuclear systems.

Two-body reactions in which neutron rich hypernuclei could be produced are the following double charge-exchange reactions:

$$
K^{-}+{ }^{A} Z \rightarrow{ }_{\Lambda}^{A}(Z-2)+\pi^{+},
$$

induced on nuclear targets by stopped $K^{-}$mesons or in flight, and

$$
\pi^{-}+{ }^{A} Z \rightarrow{ }_{\Lambda}^{A}(Z-2)+K^{+}
$$

with $\pi^{-}$mesons in flight $\left(p_{\pi^{-}}>0.89 \mathrm{GeV} / c\right)$.

The simplest description of the above reactions is a two-step process on two different protons of the same nucleus, converting them into a neutron and a $\Lambda$, with the additional condition that the final nuclear system is bound. For (1) it amounts to $K^{-} p \rightarrow \Lambda \pi^{0}$ reaction followed by $\pi^{0} p \rightarrow n \pi^{+}$or $K^{-} p \rightarrow \bar{K}^{0} n$ followed by $\bar{K}^{0} p \rightarrow \Lambda \pi^{+}$, for (2) to a $\pi^{-} p \rightarrow n \pi^{0}$ reaction followed by $\pi^{0} p \rightarrow K^{+} \Lambda$ or $\pi^{-} p \rightarrow K^{0} \Lambda$ followed by $K^{0} p \rightarrow K^{+} n$. Another mechanism is a single-step double charge exchange $m_{i}^{-} p \rightarrow \Sigma^{-} m_{f}^{+}$(where $m$ stands for meson) feeding the $\Sigma$ component coherently admixed into the final $\Lambda$ hypernuclear state. Such admixtures are essentially equivalent to invoking a second step of $\Sigma^{-} p \rightarrow \Lambda n$ conversion. These two-step processes are expected to occur at a much lower rate (reduction factor $\leqslant 10^{-2}[5]$ ) than the production of normal $\Lambda$ hypernuclei by means of the corresponding single-step two-body reactions $\left(K^{-}, \pi^{-}\right)$ and $\left(\pi^{+}, K^{+}\right)$. 
The first experimental attempt to produce neutron-rich hypernuclei by the reaction (1) with $K^{-}$at rest was carried out at KEK [6]. Upper limits were obtained for the production of ${ }_{\Lambda}^{9} \mathrm{He},{ }_{\Lambda}^{12} \mathrm{Be}$ and ${ }_{\Lambda}^{16} \mathrm{C}$ hypernuclei (on ${ }^{9} \mathrm{Be},{ }^{12} \mathrm{C}$ and ${ }^{16} \mathrm{O}$ targets respectively) in the range of $(0.6-2.0) \cdot 10^{-4} / K_{\text {stop }}^{-}$, while the theoretical predictions for ${ }_{\Lambda}^{12} \mathrm{Be}$ and ${ }_{\Lambda}^{16} \mathrm{C}$ [7] lie in the interval $\left(10^{-6}-10^{-7}\right) / K_{\text {stop }}^{-}$, which is at least one order of magnitude lower than the experimental upper limits and three orders of magnitude smaller than the standard one-step $\left(K_{\text {stop }}^{-}, \pi^{-}\right)$reaction rates on the same targets $\left(10^{-3} / K_{\text {stop }}^{-}\right)$.

Another KEK experiment [8] reported the observation of ${ }_{\Lambda}^{10} \mathrm{Li}$ in the $\left(\pi^{-}, K^{+}\right)$reaction on a ${ }^{10} \mathrm{~B}$ target with a $1.2 \mathrm{GeV} / c \pi^{-}$beam. A production cross section of $11.3 \pm 1.9 \mathrm{nb} / \mathrm{sr}$ was evaluated; the result, however, is not directly comparable with theoretical calculations [9] since no discrete structure was observed and the production cross section was integrated over the whole bound region $\left(0<B_{\Lambda}<20 \mathrm{MeV}\right)$.

A further attempt to observe neutron-rich hypernuclei by means of the reaction (1), with $K^{-}$at rest, was made at the DA $\Phi$ NE collider at LNF by the FINUDA experiment [10], on ${ }^{6} \mathrm{Li}$ and ${ }^{7} \mathrm{Li}$ targets. The limited data sample collected during the first run period of the experiment was used to estimate the production rates per stopped $K^{-}$of ${ }_{\Lambda}^{6} \mathrm{H}$ and ${ }_{\Lambda}^{7} \mathrm{H}$. The inclusive $\pi^{+}$ spectra from ${ }^{6} \mathrm{Li}$ and ${ }^{7} \mathrm{Li}$ targets were analyzed in momentum regions corresponding, through momentum and energy conservation, to $B_{\Lambda}$ values discussed in the literature. Because of the dominant contribution of the reactions

$$
\begin{aligned}
K_{\text {stop }}^{-}+p \rightarrow & \Sigma^{+}+\pi^{-} \\
& \hookrightarrow n+\pi^{+} \quad\left(\sim 130<p_{\pi^{+}}<250 \mathrm{MeV} / c\right)
\end{aligned}
$$

and

$$
\begin{aligned}
K_{\text {stop }}^{-}+p p \rightarrow & \Sigma^{+}+n \\
& \hookrightarrow n+\pi^{+} \quad\left(\sim 100<p_{\pi^{+}}<320 \mathrm{MeV} / c\right),
\end{aligned}
$$

which give the main component of the inclusive $\pi^{+}$spectra for absorption of stopped $K^{-}$mesons on nuclei, and owing to a limited statistics, only upper limits could be evaluated for $\Lambda$ hypernuclear production:

$$
\begin{aligned}
& R_{\pi^{+}}\left({ }_{\Lambda}^{6} \mathrm{H}\right)<\left(2.5 \pm 0.4_{\text {stat }_{-0.1 \text { syst }}^{+0.4}}^{+0}\right) \cdot 10^{-5} / K_{\text {stop }}^{-}, \\
& R_{\pi^{+}}\left({ }_{\Lambda}^{7} \mathrm{H}\right)<\left(4.5 \pm 0.9_{\text {stat } \left._{-0.1 \text { syst }}^{+0.4}\right) \cdot 10^{-5} / K_{\text {stop }}^{-},}\right.
\end{aligned}
$$

in addition to an upper limit determined in ${ }^{12} \mathrm{C}$ :

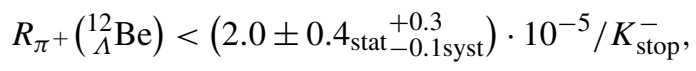

lowering by a factor $\sim 3$ the previous KEK determination [6].

In this article we present the analysis of the total data sample of the FINUDA experiment, collected from 2003 to 2007 and corresponding to a total integrated luminosity of $1156 \mathrm{pb}^{-1}$, aiming at assessing the existence of ${ }_{\Lambda}^{6} \mathrm{H}$ and determining the production rate by means of the $\left(K_{\text {stop }}^{-}, \pi^{+}\right)$reaction on ${ }^{6} \mathrm{Li}$ targets. A preliminary account of the results, reporting three clear events of ${ }_{\Lambda}^{6} \mathrm{H}$, appeared in [11].

The binding energy of ${ }_{\Lambda}^{6} \mathrm{H}$ with respect to the unstable ${ }^{5} \mathrm{H}$ core was estimated in Refs. [1,3] as $B_{\Lambda}=4.2 \mathrm{MeV}$, making ${ }_{\Lambda}^{6} \mathrm{H}$ particle stable with respect to its ${ }_{\Lambda}^{4} \mathrm{H}+2 n$ lowest threshold, as shown in Fig. 1. We recall that the binding energy $B_{\Lambda}$ of hypernucleus ${ }_{\Lambda}^{A} \mathrm{Z}$ is defined as: 


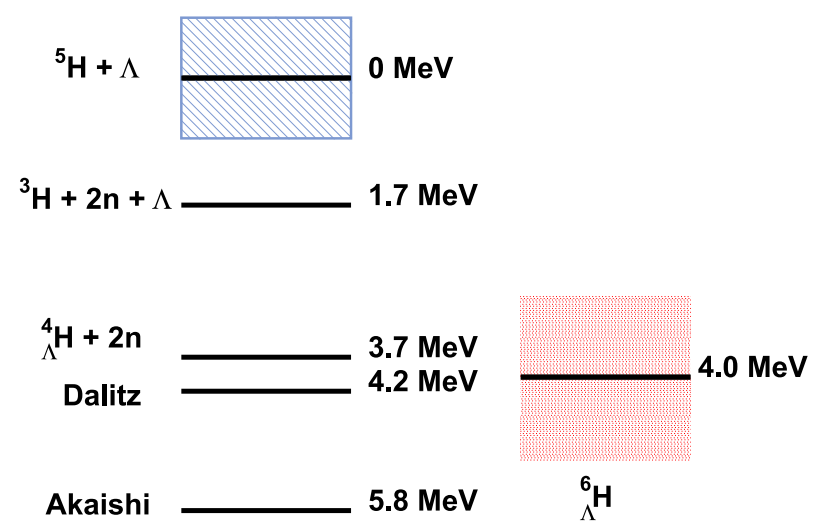

Fig. 1. Left: Binding energy scheme for a system of one proton, four neutrons and one $\Lambda$ relative to the summed mass of ${ }^{5} \mathrm{H}+\Lambda, M=5805.44 \mathrm{MeV}$, with the (blue in the web version) hatched box denoting the width of ${ }^{5} \mathrm{H}$ [12]. The two lowest horizontal lines stand for predictions from Refs. [1,13]. Right: mean value of the ${ }_{\Lambda}^{6} \mathrm{H}$ g.s. mass obtained in the present analysis jointly from production and decay; the (red in the web version) shaded box represents the error on the mass mean value obtained from the three ${ }_{\Lambda}^{6} \mathrm{H}$ events reported here.

$$
B_{\Lambda}=M_{\text {core }}+M_{\Lambda}-M_{A} Z
$$

where $M_{\text {core }}$ is the mass of the ${ }^{(A-1)} \mathrm{Z}$ core nucleus in its ground state (g.s.), as deduced from the atomic mass tables [14]. The ${ }^{5} \mathrm{H}$ nuclear core, colloquially termed "superheavy hydrogen", was observed as a broad resonance (1.9 MeV FWHM) at energy about $1.7 \mathrm{MeV}$ above the ${ }^{3} \mathrm{H}+2 n$ threshold [12]. A substantially stronger binding, $B_{\Lambda}\left({ }_{\Lambda}^{6} \mathrm{H}\right)=5.8 \mathrm{MeV}$, was predicted by Akaishi et al. [13] for the $0^{+}$g.s. on the basis of a coherent $\Lambda N-\Sigma N$ mixing model originally practised for the ${ }_{\Lambda}^{4} \mathrm{H}$ cluster [15]. This coherent $\Lambda N-\Sigma N$ mixing induces a spin-dependent $\Lambda N N$ threebody interaction which affects primarily the $0^{+}$g.s., increasing thus the $\approx 1 \mathrm{MeV} 1^{+}$excitation expected from ${ }_{\Lambda}^{4} \mathrm{H}$ to $2.4 \mathrm{MeV}$ in ${ }_{\Lambda}^{6} \mathrm{H}$. If this prediction is respected by Nature, it could imply far-reaching consequences to strange dense stellar matter.

In the next sections we describe briefly the FINUDA experimental apparatus, and the analysis technique applied to the data collected on ${ }^{6} \mathrm{Li}$ targets. We then report on three ${ }_{\Lambda}^{6} \mathrm{H}$ candidate events found by observing $\pi^{+}$mesons from production and $\pi^{-}$mesons from decay in coincidence. These events prove robust against varying the cuts selected in the analysis, and give evidence for a particle stable ${ }_{\Lambda}^{6} \mathrm{H}$. The measurement background is evaluated and the production rate of ${ }_{\Lambda}^{6} \mathrm{H}$ is estimated. We end with a brief discussion of the ${ }_{\Lambda}^{6} \mathrm{H}$ excitation spectrum as constrained by the three candidate events.

\section{Experimental apparatus}

FINUDA was a hypernuclear physics experiment installed at one of the two interaction regions of the DA $\Phi$ NE $e^{+} e^{-}$collider, the INFN-LNF $\Phi(1020)$-factory. A detailed description of the experimental apparatus can be found in Ref. [16]. The layout figured a cylindrical symmetry arrangement; here we briefly sketch its main components moving outwards from the beam axis: the interaction/target region, composed by a barrel of 12 thin scintillator slabs (TOFINO), surrounded by an octagonal array of Si microstrips (ISIM) facing eight target tiles; the tracking device, consisting of four layers of position sensitive detectors (a decagonal array of Si microstrips (OSIM), two octagonal layers of low mass drift chambers (LMDC) and a stereo system 


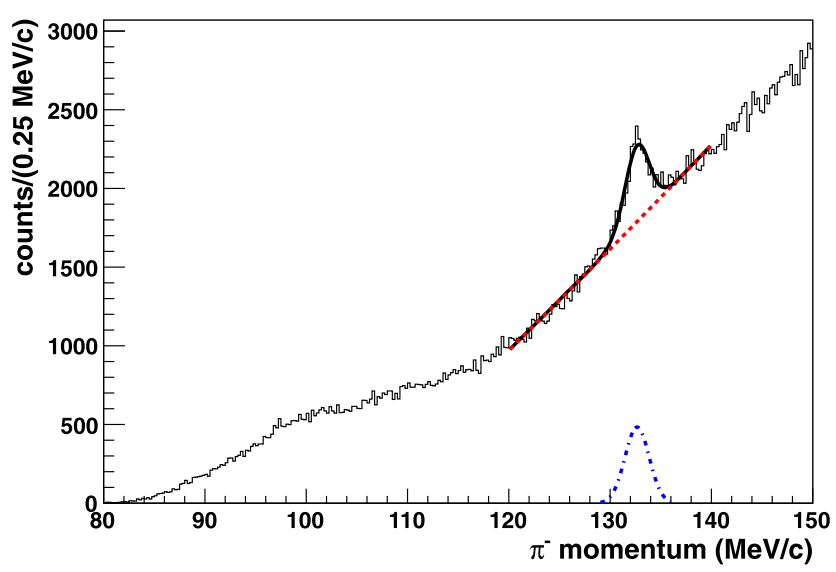

Fig. 2. Distribution of low momentum $\pi^{-}$from ${ }^{6} \mathrm{Li}$ targets. The continuous black curve represents a fit the spectrum given by the sum of a second degree polynomial [dashed (red in the web version) curve] and a gaussian function [dotdashed (blue in the web version) curve]. For more details, see text.

of straw tubes (ST)) arranged in coaxial geometry; the external time of flight detector (TOFONE), a barrel of 72 scintillator slabs. The whole apparatus was placed inside a uniform $1.0 \mathrm{~T}$ solenoidal magnetic field; the tracking volume was immersed in He atmosphere to minimize the multiple scattering effect.

The main features of the apparatus were the thinness of the target materials needed to stop the low energy $(\sim 16 \mathrm{MeV}) K^{-}$'s from the $\Phi \rightarrow K^{-} K^{+}$decay channel, the high transparency of the FINUDA tracker and the very large solid angle $(\sim 2 \pi$ sr) covered by the detector ensemble; accordingly, the FINUDA apparatus was suitable to study simultaneously the formation and the decay of $\Lambda$ hypernuclei by means of high resolution magnetic spectroscopy of the emitted charged particles.

In particular, for $\pi^{+}$with momentum $\sim 250 \mathrm{MeV} / c$ the resolution of the tracker can be evaluated by measuring the width of the momentum distribution of the monochromatic $(235.6 \mathrm{MeV} / c)$ $\mu^{+}$coming from the $K_{\mu 2}$ decay channel; for reactions occurring in the apparatus sector where ${ }^{6} \mathrm{Li}$ targets were located, it is $\sigma_{p}=(1.1 \pm 0.1) \mathrm{MeV} / c$ [17]; the precision on the absolute momentum calibration, obtained from the mean value of the same distribution, is better than $0.12 \mathrm{MeV} / c$ for the ${ }^{6} \mathrm{Li}$ targets, which corresponds to a maximum systematic uncertainty in the kinetic energy $\sigma_{T \text { sys }}\left(\pi^{+}\right)=0.1 \mathrm{MeV}$.

For $\pi^{-}$with momentum $\sim 130 \mathrm{MeV} / c$ the resolution and absolute calibration can be evaluated from the momentum distribution of the monochromatic $\pi^{-}$coming from the two-body mesonic weak decay of ${ }_{\Lambda}^{4} \mathrm{H}$, produced as hyperfragment with a formation probability of the order of $10^{-3}-10^{-2}$ per stopped $K^{-}$[18]. Fig. 2 shows the distribution for low momentum $\pi^{-}$from ${ }^{6} \mathrm{Li}$ targets, before acceptance correction; the spectrum is fitted in the $120-140 \mathrm{MeV} / c$ momentum range (continuous black curve) with the sum of a second degree polynomial, representing the background from quasi-free $\Lambda$ decay and quasi-free $\Sigma^{+}$production (dashed (red in the web version) curve in the figure), and a gaussian function representing the ${ }_{\Lambda}^{4} \mathrm{H}$ mesonic decay contribution (dot-dashed (blue in the web version) curve); the fit gives a $\chi^{2} /$ ndf $=79.1 / 74$, a mean $\mu_{p}=$ $(132.6 \pm 0.1) \mathrm{MeV} / c$ and a standard deviation $\sigma_{p}=(1.2 \pm 0.1) \mathrm{MeV} / c$ for the gaussian function, directly measuring the experimental resolution. For comparison, $p_{\pi^{-}}=(132.80 \pm 0.08) \mathrm{MeV} / c$ from $B_{\Lambda}\left({ }_{4}^{\Lambda} \mathrm{H}\right)=2.04 \pm 0.04 \mathrm{MeV}$, as determined from emulsion studies [2]; hence the absolute 


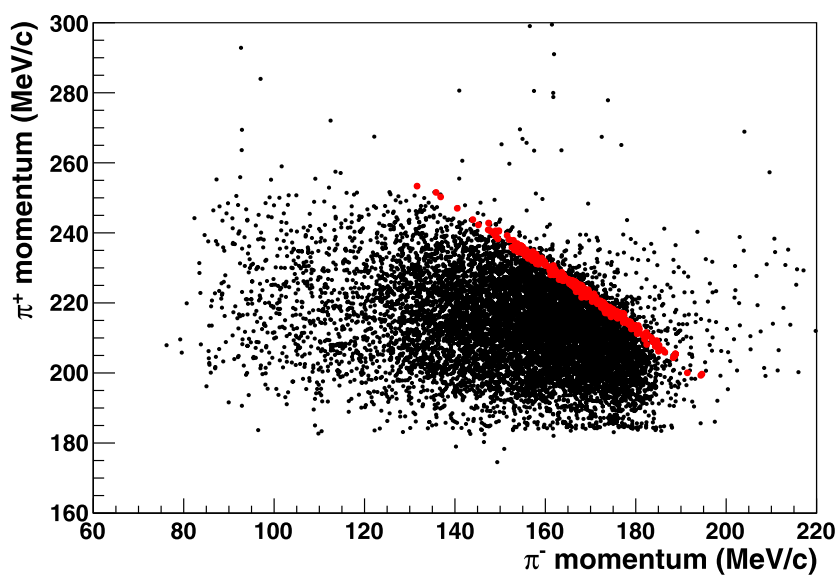

Fig. 3. $\pi^{+}$momentum vs $\pi^{-}$momentum for ${ }^{6} \mathrm{Li}$ targets. The red band dots stand for events with $T\left(\pi^{+}\right)+T\left(\pi^{-}\right)=$ 202-204 MeV. See text for more details.

uncertainty is $0.2 \mathrm{MeV} / c$ and the corresponding systematic uncertainty in the kinetic energy is then $\sigma_{T \text { sys }}\left(\pi^{-}\right)=0.14 \mathrm{MeV}$.

To perform particle identification, the information of the specific energy loss in both OSIM and the LMDC's is used; the mass identification from the time of flight system (TOFINOTOFONE) for high momentum tracks is also used. The final selection is performed by requiring the same identification from at least two different detectors.

\section{Analysis technique}

In the second data taking the statistics collected with ${ }^{6} \mathrm{Li}$ targets was improved by a factor 5 with respect to the first run. However, even with the improved statistics, we could not observe in the inclusive $\pi^{+}$spectra clear peaks that could be attributed to the two-body reaction:

$$
K_{\text {stop }}^{-}+{ }^{6} \mathrm{Li} \rightarrow{ }_{\Lambda}^{6} \mathrm{H}+\pi^{+} \quad\left(p_{\pi^{+}} \sim 252 \mathrm{MeV} / c\right) .
$$

Exploiting the increased statistics, we tried then to reduce the background overwhelming the events from reaction (9) by examining the spectra of $\pi^{+}$in coincidence with the $\pi^{-}$coming from the mesonic decay of ${ }_{\Lambda}^{6} \mathrm{H}$ :

$$
{ }_{\Lambda}^{6} \mathrm{H} \rightarrow{ }^{6} \mathrm{He}+\pi^{-} \quad\left(p_{\pi^{-}} \sim 130-140 \mathrm{MeV} / c\right) .
$$

The branching ratio for (10) is expected to be about $50 \%$ taking into account the value measured for the analogous decay ${ }_{\Lambda}^{6} \mathrm{H} \rightarrow{ }^{4} \mathrm{He}+\pi^{-}[18] .\left(\pi^{+}, \pi^{-}\right)$coincidence events, associated with $K^{-}$'s stopped in the ${ }^{6} \mathrm{Li}$ targets, were thus considered; only reaction (3) contributes to the background of this sample.

We examined thus the two-dimensional raw spectrum of $\pi^{+}$versus $\pi^{-}$momentum, shown in Fig. 3, in order to recognize possible enhancements due to occurrence of the reactions (9) and (10) in sequence. The low statistics and the strong background prevented us from finding statistically significant accumulations of events in the plot arising from a bound ${ }_{\Lambda}^{6} \mathrm{H}$.

In order to isolate the events due to the possible formation of a bound ${ }_{\Lambda}^{6} \mathrm{H}$, we considered energy conservation for both reactions (9) and (10). Momentum conservation is automatically 
ensured by the fact that both reactions of formation (9) and decay (10) occur at rest. The stopping time of ${ }_{\Lambda}^{6} \mathrm{H}$ in the material is indeed shorter than its lifetime.

For (9) we may write explicitly:

$$
M\left(K^{-}\right)+3 M(p)+3 M(n)-B\left({ }^{6} \mathrm{Li}\right)=M\left({ }_{\Lambda}^{6} \mathrm{H}\right)+T\left({ }_{\Lambda}^{6} \mathrm{H}\right)+M\left(\pi^{+}\right)+T\left(\pi^{+}\right),
$$

in which, in obvious notation, $M$ stands for a particle mass, $T$ - its kinetic energy, and $B\left({ }^{6} \mathrm{Li}\right)-$ the binding energy of ${ }^{6} \mathrm{Li}$. For (10) we may write:

$$
M\left({ }_{\Lambda}^{6} \mathrm{H}\right)=2 M(p)+4 M(n)-B\left({ }^{6} \mathrm{He}\right)+T\left({ }^{6} \mathrm{He}\right)+M\left(\pi^{-}\right)+T\left(\pi^{-}\right),
$$

in the same notation as above. Combining Eqs. (11) and (12) in order to eliminate $M\left({ }_{\Lambda}^{6} \mathrm{H}\right)$, we get the following equation:

$$
\begin{aligned}
T\left(\pi^{+}\right)+T\left(\pi^{-}\right)= & M\left(K^{-}\right)+M(p)-M(n)-2 M(\pi) \\
& -B\left({ }^{6} \mathrm{Li}\right)+B\left({ }^{6} \mathrm{He}\right)-T\left({ }^{6} \mathrm{He}\right)-T\left({ }_{\Lambda}^{6} \mathrm{H}\right) .
\end{aligned}
$$

All the terms on the right-hand side are either known constants or quantities that can be evaluated from momentum and energy conservation, except for $T\left({ }_{\Lambda}^{6} \mathrm{H}\right)\left(T\left({ }^{6} \mathrm{He}\right)\right)$ that depends explicitly (implicitly) on the unknown value of $B_{\Lambda}\left({ }_{\Lambda}^{6} \mathrm{H}\right)$. A variation of $B_{\Lambda}\left({ }_{\Lambda}^{6} \mathrm{H}\right)$ between 0 and $6 \mathrm{MeV}$ introduces a change of $\sim 0.3 \mathrm{MeV}$ in the kinetic energy $T\left({ }_{\Lambda}^{6} \mathrm{H}\right)$ in (11), corresponding to a sensitivity of $50 \mathrm{keV}$ per $\mathrm{MeV}$ of $B_{\Lambda}\left({ }_{\Lambda}^{6} \mathrm{H}\right)$, and a change of $\sim 0.2 \mathrm{MeV}$ in $T\left(\pi^{+}\right)+T\left(\pi^{-}\right)$in (13), corresponding to a sensitivity of $30 \mathrm{keV}$ per $\mathrm{MeV}$ of $B_{\Lambda}\left({ }_{\Lambda}^{6} \mathrm{H}\right)$. These variations are much lower than the experimental energy resolutions for $\pi^{+}(250 \mathrm{MeV} / c)$ and $\pi^{-}(130 \mathrm{MeV} / c): \sigma_{T}\left(\pi^{+}\right)=0.96 \mathrm{MeV}$ and $\sigma_{T}\left(\pi^{-}\right)=0.84 \mathrm{MeV}$. The FINUDA energy resolution for a $\left(\pi^{+}, \pi^{-}\right)$pair in coincidence is therefore $\sigma_{T}=\sqrt{\sigma_{T \exp }^{2}+\sigma_{T \text { sys }}^{2}}=1.3 \mathrm{MeV}$, where $\sigma_{T \exp }=\sqrt{0.96^{2}+0.84^{2}}=1.3 \mathrm{MeV}$ is the total experimental energy resolution and $\sigma_{T \text { sys }}=\sqrt{\sigma_{T \text { sys }}\left(\pi^{+}\right)^{2}+\sigma_{T \text { sys }}\left(\pi^{-}\right)^{2}}=0.17 \mathrm{MeV}$ is the total systematic error on energy. To be definite, we assume a value of $B_{\Lambda}\left({ }_{\Lambda}^{6} \mathrm{H}\right)=5 \mathrm{MeV}$, halfway between the conservative estimate of 4.2 MeV [1,3] and Akaishi's prediction of 5.8 MeV [13]. The r.h.s. of Eq. (13) assumes then a value of $T\left(\pi^{+}\right)+T\left(\pi^{-}\right)=203.0 \pm 1.3 \mathrm{MeV}$.

We considered then the raw spectrum of the total kinetic energy, $T\left(\pi^{+}\right)+T\left(\pi^{-}\right)$, for the coincidence events, shown in Fig. 4. Events in the summed energy distribution were selected in the region $(203 \pm 1) \mathrm{MeV}$, indicated by the (red in the web version) filling in the figure. The halfwidth of the interval corresponds to $\sim 77 \%$ of the FINUDA total energy resolution; the value was chosen as a compromise between the strong requirement of reducing the contamination from background reactions, as will be discussed in more detail in the following, and the plight for reasonable statistics, leading to application of a selection narrower than the experimental resolution. The selected events are represented by red dots in Fig. 3.

The raw distributions of $p_{\pi^{+}}$and $p_{\pi^{-}}$for the events selected are shown in Fig. 5 by the continuous line histogram, falling off to zero at $p_{\pi^{+}}=245 \mathrm{MeV} / c$ in the higher momentum region, and at $p_{\pi^{-}}=145 \mathrm{MeV} / c$ in the lower momentum region. These limiting values, when inserted in Eqs. (9) and (10) for two-body kinematics ${ }_{\Lambda}^{6} \mathrm{H}$ production from rest and decay at rest, yield ${ }_{\Lambda}^{6} \mathrm{H}$ mass values higher than the total mass of both $\left(\Lambda+{ }^{3} \mathrm{H}+2 n\right)$ and $\left(\Lambda+{ }^{5} \mathrm{H}\right)$ thresholds marked in Fig. 1. A ${ }_{\Lambda}^{6} \mathrm{H}$ mass equal to the mass of its lowest particle stability threshold ${ }_{\Lambda}^{4} \mathrm{H}+2 n$ corresponds to values of $p_{\pi^{+}}=251.9 \mathrm{MeV} / c$ and $p_{\pi^{-}}=135.6 \mathrm{MeV} / c$. A genuinely bound ${ }_{\Lambda}^{6} \mathrm{H}$ system, therefore, requires that pion momenta satisfying $p_{\pi^{+}}>251.9 \mathrm{MeV} / c$ and 


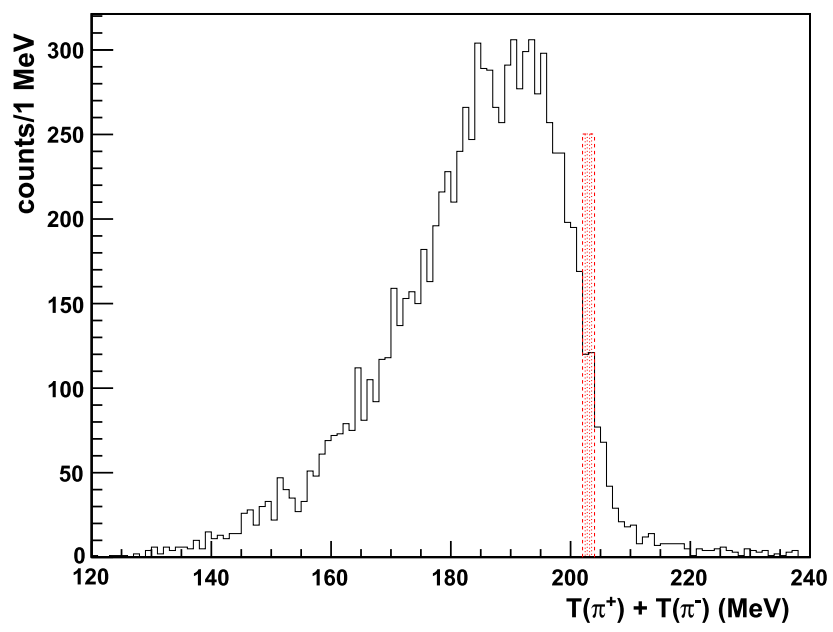

Fig. 4. Distribution of raw total kinetic energy $T\left(\pi^{+}\right)+T\left(\pi^{-}\right)$for $\left(\pi^{+}, \pi^{-}\right)$coincidence events from ${ }^{6} \mathrm{Li}$ targets. The (red in the web version) shaded vertical bar represents the cut $T\left(\pi^{+}\right)+T\left(\pi^{-}\right)=202-204 \mathrm{MeV}$.

$p_{\pi^{-}}<135.6 \mathrm{MeV} / c$ are selected. The cuts actually applied in the analysis of the data, $p_{\pi^{+}}=$ (250-255) MeV/c and $p_{\pi^{-}}=(130-137) \mathrm{MeV} / c$, as marked by the (blue) shaded vertical bars in Fig. 5, allow for a wide range of ${ }_{\Lambda}^{6} \mathrm{H}$ masses from the $\left(\Lambda+{ }^{3} \mathrm{H}+2 n\right)$ threshold, about $2 \mathrm{MeV}$ in the ${ }_{\Lambda}^{6} \mathrm{H}$ continuum, down to $B_{\Lambda}\left({ }_{\Lambda}^{6} \mathrm{H}\right) \geqslant 6 \mathrm{MeV}$, somewhat below the mass predicted by Akaishi [13]. These cuts do not exclude completely an eventual contribution from the production and decay of ${ }_{\Lambda}^{4} \mathrm{H}+2 n$, of a weight which is anyway negligible, as discussed in the next section.

\section{Results}

Three events, out of a total number of $\sim 2.7 \cdot 10^{7} \mathrm{~K}^{-}$detected at stop on the ${ }^{6} \mathrm{Li}$ targets, satisfy the final requirements, $T\left(\pi^{+}\right)+T\left(\pi^{-}\right)=202-204 \mathrm{MeV}, p_{\pi^{+}}=250-255 \mathrm{MeV} / c$ and $p_{\pi^{-}}=130-137 \mathrm{MeV} / c$. These events, within the (red in the web version) shaded rectangle on the 1.h.s. of Fig. 6, are candidates for ${ }_{\Lambda}^{6} \mathrm{H}$. The $\pi^{+}$momenta which this rectangle encompasses go up from a value corresponding to the $\left(\Lambda+{ }^{3} \mathrm{H}+2 n\right)$ threshold to a value corresponding to the binding energy predicted by Akaishi, whereas the $\pi^{-}$momenta which the rectangle encompasses go down from a value corresponding to the same $\left(\Lambda+{ }^{3} \mathrm{H}+2 n\right)$ threshold to about $2 \sigma\left(p_{\pi^{-}}\right)$ below the value predicted by Akaishi [13].

Different choices of $T\left(\pi^{+}\right)+T\left(\pi^{-}\right)$interval widths $(2-6 \mathrm{MeV})$ and position (center in 202-204 MeV) and of $p_{\pi^{+}} / p_{\pi^{-}}$interval widths (5-10 and 8-15 MeV/c) with fixed limits at 250 and $137 \mathrm{MeV} / c$ respectively to exclude the unbound region, affect the populations of the corresponding single spectra but not the coincidence spectrum. As an example, in Fig. 6 a comparison is made between the $\left(p_{\pi^{+}}, p_{\pi^{-}}\right)$plots satisfying the actual selection $T\left(\pi^{+}\right)+T\left(\pi^{-}\right)=202-204 \mathrm{MeV}$ (l.h.s.), and similar plots admitting a wider selection range $T\left(\pi^{+}\right)+T\left(\pi^{-}\right)=200-206 \mathrm{MeV}$ (r.h.s.). The global population increases for the wider cut, as expected, but the events that satisfy simultaneously also the separate selections imposed on $p_{\pi^{+}}$ and $p_{\pi^{-}}$(shaded rectangles in the upper left part of the plots) remain the same. A similar stability is not observed in the opposite corner of the plots where, on top of the events already there on the left plot, five additional events appear on the right plot upon extending the cut. Quanti- 

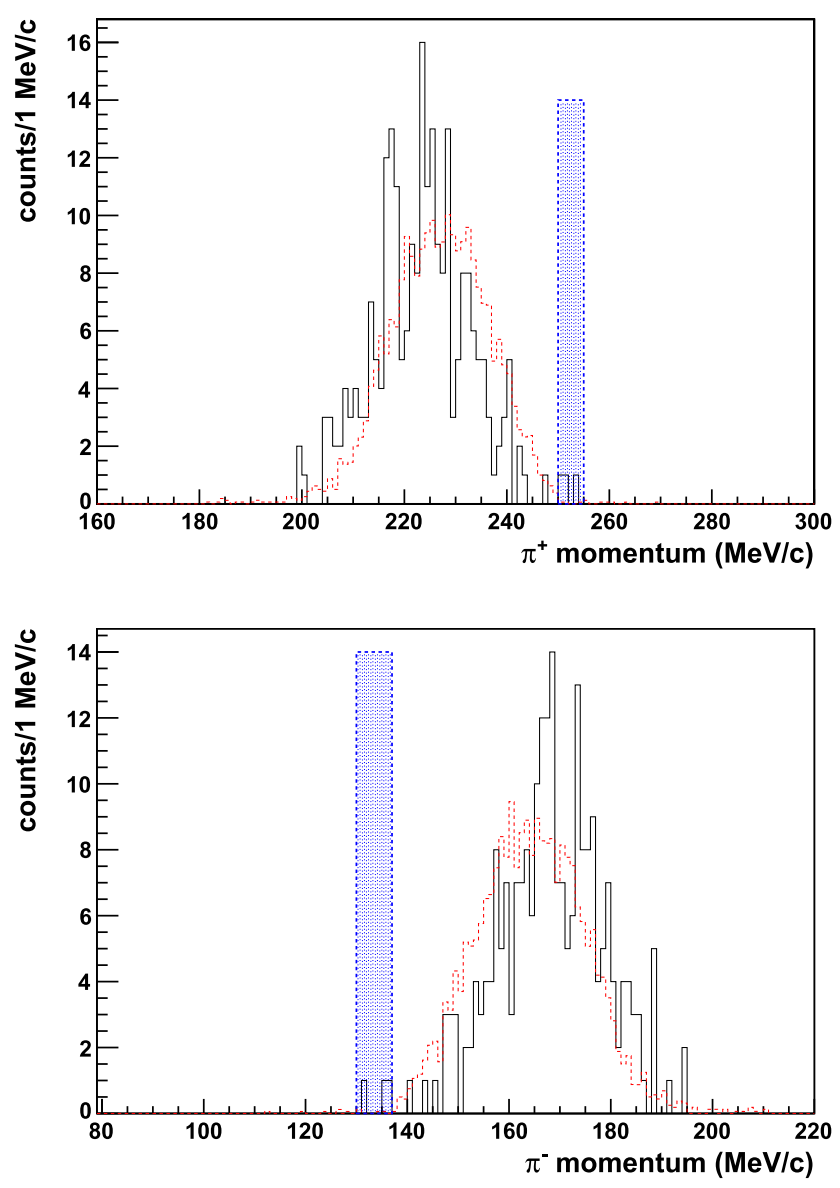

Fig. 5. Continuous histograms: distribution of $\pi^{+}$(upper part) and $\pi^{-}$(lower part) momenta for $\left(\pi^{+}, \pi^{-}\right.$) coincidence events with $T\left(\pi^{+}\right)+T\left(\pi^{-}\right)=202-204 \mathrm{MeV}$ from ${ }^{6} \mathrm{Li}$ targets before acceptance correction. The (blue in the web version) shaded vertical bars indicate the final selection regions. Dashed (red in the web version) histograms represent the $\Sigma^{+}$background spectra: see text for more details.

tatively, fitting the projected $\pi^{ \pm}$distributions of the 1.h.s. of Fig. 6 by gaussians, an excess of three events in both $p_{\pi^{ \pm}}$distributions is invariably found, corresponding to the shaded (red in the web version) rectangle. The probability for the three events to belong to the fitted gaussian (background) distribution is less than $0.5 \%$ in both cases. It is possible, moreover, to see directly from the two-dimensional plots that variations of the independent momentum selections do not produce any effect. Systematic errors due to the applied analysis selection are thus ruled out.

It is also worth noticing that the tight momentum cuts imposed on the $\left(\pi^{+}, \pi^{-}\right)$coincidence events allow to eliminate completely any contamination due to possible $\pi^{-} / e^{-}$misidentification. Furthermore, $\mu^{+}$'s from $K_{\mu 2}^{+}$decay are clearly separated from $\pi^{+}$'s coming from the opposite $K^{-}$interaction vertex. Fig. 7 shows a front view of one of the three events, as reconstructed by FINUDA.

By evaluating event by event the corresponding ${ }_{\Lambda}^{6} \mathrm{H}$ mass from both production (9) and decay (10) reactions, the mass values listed in Table 1 are obtained. A mean value for each event 

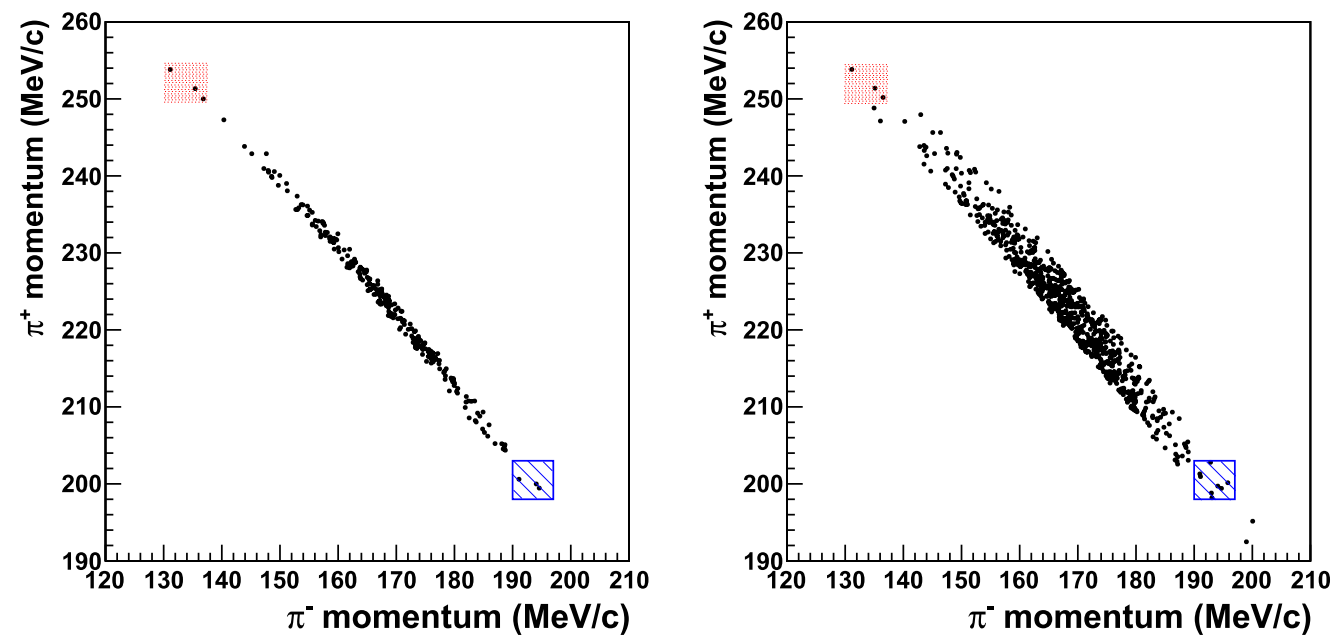

Fig. 6. $\pi^{+}$momentum vs $\pi^{-}$momentum for ${ }^{6} \mathrm{Li}$ target events with $T\left(\pi^{+}\right)+T\left(\pi^{-}\right)=202-204 \mathrm{MeV}$ (1.h.s.) and with $T\left(\pi^{+}\right)+T\left(\pi^{-}\right)=200-206 \mathrm{MeV}$ (r.h.s.). The shaded (red in the web version) rectangles on each side consist of a subset of events with $p_{\pi^{+}}=250-255 \mathrm{MeV} / c$ and $p_{\pi^{-}}=130-137 \mathrm{MeV} / c$. The hatched (blue in the web version) rectangles on each side are symmetric subsets of events to those in the shaded rectangles.
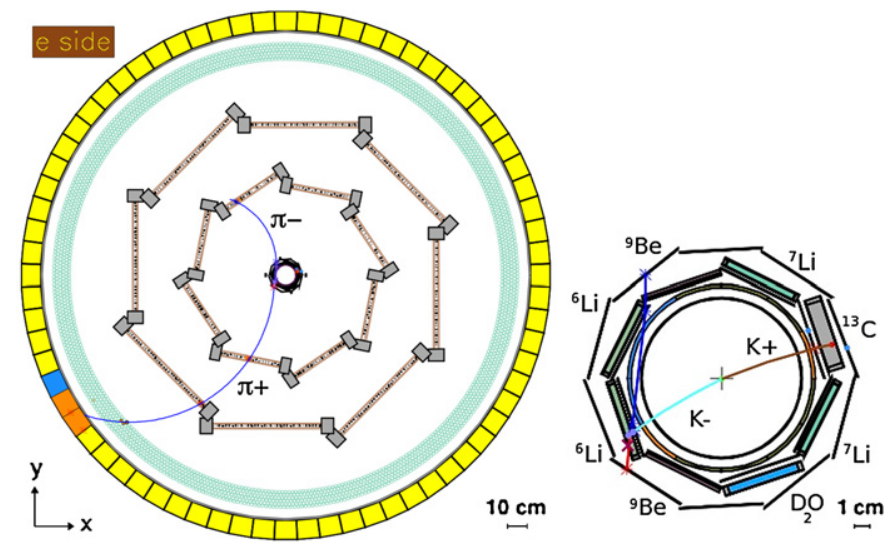

Fig. 7. Left: front view of one of the ${ }_{\Lambda}^{6} \mathrm{H}$ candidate events reconstructed by FINUDA where a $\left(\pi^{+}, \pi^{-}\right)$pair emerges from a ${ }^{6} \mathrm{Li}$ target and crosses the spectrometer. Right: expanded view of the target region for the same event where the $K^{-}$track stops in a ${ }^{6} \mathrm{Li}$ target.

Table 1

Kinematical properties and ${ }_{\Lambda}^{6} \mathrm{H}$ mass, $M\left({ }_{\Lambda}^{6} \mathrm{H}\right)$, of the three ${ }_{\Lambda}^{6} \mathrm{H}$ candidate events from production (9) and decay (10) reactions. Listed in the last two columns are the mean and the difference of the production and decay masses. $T_{\text {tot }}$ indicates the total $\pi^{ \pm}$kinetic energy $T\left(\pi^{+}\right)+T\left(\pi^{-}\right)$.

\begin{tabular}{lllllll}
\hline $\begin{array}{l}T_{\text {tot }} \\
(\mathrm{MeV})\end{array}$ & $\begin{array}{l}p_{\pi^{+}} \\
(\mathrm{MeV} / c)\end{array}$ & $\begin{array}{l}p_{\pi^{-}} \\
(\mathrm{MeV} / c)\end{array}$ & $\begin{array}{l}M\left({ }_{\Lambda}^{6} \mathrm{H}\right) \\
\text { prod. }(\mathrm{MeV})\end{array}$ & $\begin{array}{l}M\left({ }_{\Lambda}^{6} \mathrm{H}\right) \\
\text { decay }(\mathrm{MeV})\end{array}$ & $\begin{array}{l}M\left({ }_{\Lambda}^{6} \mathrm{H}\right) \\
\text { mean }(\mathrm{MeV})\end{array}$ & $\begin{array}{l}\Delta M\left({ }_{\Lambda}^{6} \mathrm{H}\right) \\
(\mathrm{MeV})\end{array}$ \\
\hline $202.6 \pm 1.3$ & $251.3 \pm 1.1$ & $135.1 \pm 1.2$ & $5802.33 \pm 0.96$ & $5801.41 \pm 0.84$ & $5801.87 \pm 0.96$ & $0.92 \pm 1.28$ \\
$202.7 \pm 1.3$ & $250.1 \pm 1.1$ & $136.9 \pm 1.2$ & $5803.45 \pm 0.96$ & $5802.73 \pm 0.84$ & $5803.09 \pm 0.96$ & $0.72 \pm 1.28$ \\
$202.1 \pm 1.3$ & $253.8 \pm 1.1$ & $131.2 \pm 1.2$ & $5799.97 \pm 0.96$ & $5798.66 \pm 0.84$ & $5799.32 \pm 0.96$ & $1.31 \pm 1.28$ \\
\hline
\end{tabular}


jointly from production and decay was also evaluated, with an error given by the highest of the two uncertainties, $0.96 \mathrm{MeV}$. For the global mean mass value of the three events we then find $\bar{M}\left({ }_{\Lambda}^{6} \mathrm{H}\right)=(5801.43 \pm 0.55) \mathrm{MeV}$, where the uncertainty $0.55=0.96 / \sqrt{3} \mathrm{MeV}$ reflects the uncertainty assigned to each event. This global mass uncertainty, however, is considerably smaller than the mean-mass spread of the three events. We therefore decided to relax the assigned uncertainty by calculating it from the spread of the three mean mass values, which yields

$$
\bar{M}\left({ }_{\Lambda}^{6} \mathrm{H}\right)=5801.4 \pm 1.1 \mathrm{MeV},
$$

with uncertainty larger than the $0.96 \mathrm{MeV}$ and $0.84 \mathrm{MeV}$ measurement uncertainties in production and decay respectively. The standard deviation of this uncertainty is $0.55 \mathrm{MeV}$ which together with $\sigma=1.1 \mathrm{MeV}$ is still short of the $2.11 \mathrm{MeV}$ deviation of the third event mean mass from the mean mass value. This observation could indicate some irregularity in the reconstruction of the third event. To regain confidence, each one of the three events was checked visually for irregularities but none was found. The third event, in particular, is shown in Fig. 7.

Listed in the last column of Table 1 are values of $\Delta M\left({ }_{\Lambda}^{6} \mathrm{H}\right)$, defined as the difference between the ${ }_{\Lambda}^{6} \mathrm{H}$ mass values obtained from production and from decay. The mass values obtained from production are systematically higher than those from decay by

$$
\Delta M\left({ }_{\Lambda}^{6} \mathrm{H}\right)=0.98 \pm 0.74 \mathrm{MeV},
$$

where the uncertainty is evaluated from the $1.3 \mathrm{MeV}$ uncertainty for $T\left(\pi^{+}\right)+T\left(\pi^{-}\right)$from which each of the mass differences is directly determined. Unlike the mean $M\left({ }_{\Lambda}^{6} \mathrm{H}\right)$ mass value, the spread of the production vs decay mass differences is well within $1 \sigma$. A possible physical origin of the $\Delta M\left({ }_{\Lambda}^{6} \mathrm{H}\right)$ systematics is discussed in a subsequent section.

The mean mass value (14) corresponds to a ${ }_{\Lambda}^{6} \mathrm{H}$ binding energy $B_{\Lambda}=(4.0 \pm 1.1) \mathrm{MeV}$ with respect to the $\left(\Lambda+{ }^{5} \mathrm{H}\right)$ threshold, and to $B_{\Lambda}=(0.3 \pm 1.1) \mathrm{MeV}$ with respect to the lowest threshold $\left({ }_{\Lambda}^{4} \mathrm{H}+2 n\right)$. The ${ }_{\Lambda}^{6} \mathrm{H}$ mean mass value and its uncertainty are indicated on the r.h.s. of Fig. 1 with respect to the various thresholds and predictions shown on the 1.h.s. of the figure.

\section{Background estimation}

Before discussing the physical interpretation of the above results, it is mandatory to check carefully that the three observed events do not arise from physical or instrumental backgrounds that could affect the data. Concerning the physical backgrounds, a complete simulation has been performed of possible $K_{\text {stop }}^{-}$absorption reactions on both single nucleons and pairs of strongly correlated nucleons that lead to the formation and decay of $\Lambda$ and $\Sigma$ hyperons. Of these reactions, only the following chain leads to $\left(\pi^{+}, \pi^{-}\right)$coincidences in the same momentum ranges corresponding to the production and mesonic decay of ${ }_{\Lambda}^{6} \mathrm{H}$ and which are respected by the three candidate events:

$$
\begin{aligned}
K_{\text {stop }}^{-}+{ }^{6} \mathrm{Li} \rightarrow & \Sigma^{+}+{ }^{4} \mathrm{He}+n+\pi^{-} \quad\left(p_{\pi^{-}} \leqslant 190 \mathrm{MeV} / c\right) \\
& \hookrightarrow n+\pi^{+} \quad\left(p_{\pi^{+}} \leqslant 282 \mathrm{MeV} / c\right) .
\end{aligned}
$$

This reaction chain has been studied by means of the FINUDA simulation program fully reproducing the apparatus geometry, detection efficiency and the trigger efficiency. The interaction of $K^{-}$with the target nucleus has been simulated with two different approaches. In the first approach, the quasi-free approximation was adopted for the interaction of the $K^{-}$with a proton of the target nucleus, $K_{\text {stop }}^{-}+p \rightarrow \Sigma^{+}+\pi^{-}$, taking into account the nucleon Fermi motion; 
the residual nucleus was considered as a spectator and the notation " ${ }^{4} \mathrm{He}+n$ " is just a label to indicate that the system is highly particle unstable. Pions arising from (16) were processed by the pattern recognition and reconstruction programs of FINUDA as real data. In common with all simulated reaction chains, the simulated events were then submitted to the same quality cuts and to the same selections criteria applied in the data analysis. Three events were found out of a total of $2.2 \cdot 10^{7} \mathrm{~K}^{-}$mesons simulated to stop on ${ }^{6} \mathrm{Li}$ targets and forced to undergo the (16) "quasi-free" reaction chain with a probability of 1 . Taking into account the number of actual $K^{-}$ mesons stopped on ${ }^{6} \mathrm{Li}$ targets, the branching fraction for the $K_{\text {stop }}^{-}+p \rightarrow \Sigma^{+}+\pi^{-}$reaction on nuclei measured on ${ }^{12} \mathrm{C}$ [19] and on ${ }^{4} \mathrm{He}$ [20], (0.159 \pm 0.012$)$ evaluated as a weighted mean, the $\Sigma^{+}+n \rightarrow \Lambda+p$ conversion probability [21], $(0.45 \pm 0.04)$, and the $\Sigma^{+} \rightarrow n+\pi^{+}$decay branching ratio, $(0.483 \pm 0.003)$, an expected $\Sigma^{+}$background of $0.15 \pm 0.09$ events on ${ }^{6} \mathrm{Li}$ targets is obtained.

In a second approach, the interaction of $K^{-}$mesons with the target nucleus as a whole was considered, applying directly the 4-body kinematics to (16). Five events were found out of a total of $2.7 \cdot 10^{7} \mathrm{~K}^{-}$mesons simulated to stop on ${ }^{6} \mathrm{Li}$ targets and forced to undergo the (16) "4-body" reaction chain with a probability of 1 . Taking into account the same normalization factors used for the "quasi-free" approach, an expected $\Sigma^{+}$reaction chain background of $0.20 \pm 0.11$ events on ${ }^{6} \mathrm{Li}$ targets was obtained under the hypothesis that $\Sigma^{+}$production on ${ }^{6} \mathrm{Li}$ in this approach always gives a recoiling ${ }^{4} \mathrm{He}$ nucleus. Final states corresponding to further fragmentation of the ${ }^{6} \mathrm{Li}$ target nucleus, such as $K_{\text {stop }}^{-}+{ }^{6} \mathrm{Li} \rightarrow \Sigma^{+}+{ }^{3} \mathrm{He}+n+n+\pi^{-}$, give weaker background contribution, owing to the requirements imposed on $T\left(\pi^{+}\right)+T\left(\pi^{-}\right)(<180 \mathrm{MeV}$ for final states of the $\Sigma^{+}$production reaction with more than 4 bodies) and on the $\pi^{+}$and $\pi^{-}$momenta.

We also considered the distortion of Eq. (16) reaction chain spectra due to the ${ }^{4} \mathrm{He}+n$ final state interaction leading to ${ }^{5} \mathrm{He}$, a resonance centered at $\sim 0.8 \mathrm{MeV}$ above the ${ }^{4} \mathrm{He}+n$ threshold with $\Gamma=1.36 \mathrm{MeV}$ [22]. To this end we required that once the ${ }^{4} \mathrm{He}$ and neutron momenta generated by the 4-body phase space simulation corresponded to the formation of the ${ }^{5} \mathrm{He}$ resonance, the momenta of the remaining particles, $\Sigma^{+}$and $\pi^{-}$, should be modified accordingly. We passed then these modified phase space distributions through the selection criteria described above and found variation of less than $1 \%$ in the background value evaluated for a $100 \%{ }^{4} \mathrm{He}+n$ final state.

In Fig. 8 the experimental $T\left(\pi^{+}\right)+T\left(\pi^{-}\right)$spectrum is shown together with spectra obtained from the "quasi-free" and "4-body" simulations of the (16) process: the simulated spectra were normalized to the area of the experimental distribution. As may be seen, the "quasi-free" spectrum (dashed (blue in the web version) histogram) reproduces the experimental distribution better than the "4-body" (dotted (violet in the web version) histogram), but exhibits a too sharp decrease in the 200-210 MeV region and underestimates the low energy tail. To obtain a satisfactory description, a fit of the experimental spectrum was performed with fractions of the two simulated templates; a standard likelihood fitting method, using Poisson statistics, was applied in which both data and Monte Carlo statistical uncertainties were taken into account [23]. Particular care was devoted to the description of the $200-210 \mathrm{MeV}$ slope, where the selection of the ${ }_{\Lambda}^{6} \mathrm{H}$ candidate events is made. The continuous (black) histogram in Fig. 8 represents the best fit to the 180-220 MeV region; the resulting fractions are $0.743 \pm 0.019$ and $0.257 \pm 0.017$ for the "quasi-free" and "4-body" templates respectively, with a $\chi^{2} /$ ndf $=40.0 / 39$. We note that varying the width of the fit region from $180-220 \mathrm{MeV}$ to $130-220 \mathrm{MeV}$ spoils the fit, increasing the $\chi^{2} /$ ndf value by a factor of $\sim 3.8$, while the fractions of the two templates change by less than 0.025 , corresponding to $1.3-1.5 \sigma$.

Back in Fig. 5, the dashed (red in the web version) histograms represent the separate $p_{\pi^{+}}$and $p_{\pi^{-}}$distributions obtained by adopting the above fractions to the events successfully simulated 


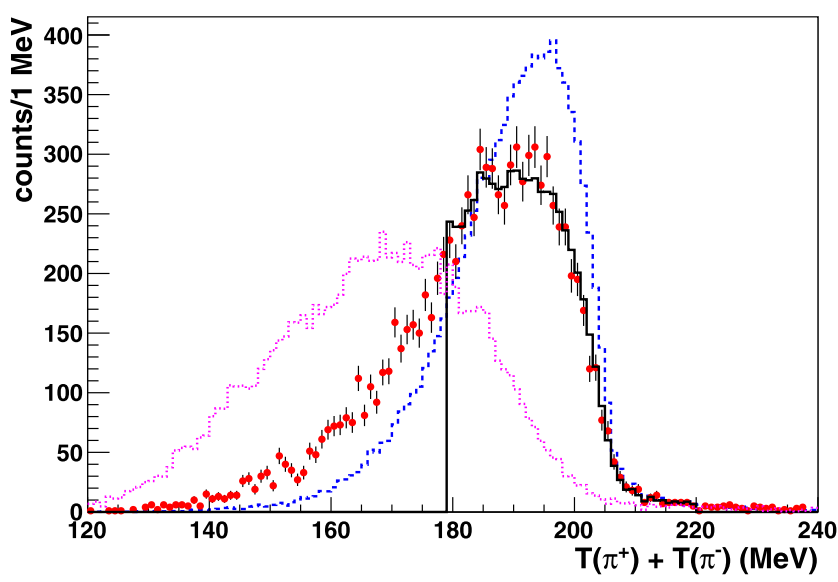

Fig. 8. $T\left(\pi^{+}\right)+T\left(\pi^{-}\right)$distributions; (red in the web version) points: experimental data; (blue in the web version) dashed histogram: "quasi-free" simulation; (violet in the web version) dotted histogram: "4-body" simulation; (black) continuous histogram: best fit to the data with fractions of the simulated templates. The simulated distributions have been normalized to the experimental distribution area. For more details see text.

within the two approaches discussed above and satisfying the cut $T\left(\pi^{+}\right)+T\left(\pi^{-}\right)=202-$ $204 \mathrm{MeV}$. Only a qualitative agreement is reached with the experimental, low statistics distributions. The estimated background spectra are shifted from the experimental ones toward higher momentum for the $\pi^{+}$spectrum and toward lower momentum for the $\pi^{-}$spectrum. The difference is significant over the statistical fluctuation. However, these shifts cause overestimates of the background counts when the tails of the simulated distributions are used for an estimate. It is thus possible to conclude that the background estimate is safe in spite of the slight deviations noted here. In particular, in the ${ }_{\Lambda}^{6} \mathrm{H}$ selected regions, indicated by shaded (blue in the web version) vertical bars, the contribution due to the $\Sigma^{+}$background for events satisfying also the cuts on $p_{\pi^{+}}$and $p_{\pi^{-}}$in coincidence corresponds to $0.16 \pm 0.07$ events on ${ }^{6} \mathrm{Li}$ targets (BGD1).

Another reaction chain capable of providing background events is

$$
\begin{aligned}
K_{\text {stop }}^{-}+{ }^{6} \mathrm{Li} \rightarrow & { }_{\Lambda}^{4} \mathrm{H}+2 n+\pi^{+} \quad\left(p_{\pi^{+}} \leqslant 252 \mathrm{MeV} / c\right) \\
& \hookrightarrow{ }^{4} \mathrm{He}+\pi^{-} \quad\left(p_{\pi^{-}} \sim 132.8 \mathrm{MeV} / c\right) .
\end{aligned}
$$

The momentum of the ${ }_{\Lambda}^{4} \mathrm{H}$ decay $\pi^{-}$is close to the momentum of the $\pi^{-}$from the two-body decay of ${ }_{\Lambda}^{6} \mathrm{H}, p_{\pi^{-}} \sim 134 \mathrm{MeV} / c$, evaluated assuming $B_{\Lambda}=5 \mathrm{MeV}$ which is halfway between the two theoretical estimates exhibited in Fig. 1.

The probability of having background contribution from this reaction chain was evaluated taking into account the phase space fraction of the reaction (17) available for $\pi^{+}$'s satisfying the momentum selection $p_{\pi^{+}}=250-255 \mathrm{MeV} / c, 4 \cdot 10^{-6}$, and the probability for a $K_{\text {stop }}^{-}$to produce a ${ }_{\Lambda}^{4} \mathrm{H}$ accompanied by a $\pi^{+}$on a ${ }^{6} \mathrm{Li}$ target. In Ref. [18] the probability of producing ${ }_{\Lambda}^{4} \mathrm{H}$ on ${ }^{7} \mathrm{Li}$ targets was reported to be $(3.0 \pm 0.4) \cdot 10^{-2} / K_{\text {stop }}^{-}$and, furthermore, the probability of producing it together with a charged pion was indicated to be $0.49 \pm 0.08$. Using these values, the formation probability of ${ }_{\Lambda}^{4} \mathrm{H}+\pi^{ \pm}$on ${ }^{6} \mathrm{Li}$ target, the closest isotope of ${ }^{7} \mathrm{Li}$, was evaluated to be $(1.47 \pm$ $0.15) \cdot 10^{-2} / K_{\text {stop }}^{-}$. In addition, a branching ratio 0.49 [18] for the two-body decay ${ }_{\Lambda}^{4} \mathrm{H} \rightarrow{ }^{4} \mathrm{He}+$ $\pi^{-}$has to be included. A total probability of $(2.8 \pm 0.5) \cdot 10^{-8}$ is obtained. From this value, taking into account the global efficiency of FINUDA (acceptance, reconstruction and analysis cuts) it is 
possible to evaluate a background of $0.05 \pm 0.01$ reconstructed events for the $2.7 \cdot 10^{7} K^{-}$mesons stopped on the ${ }^{6} \mathrm{Li}$ targets. It should be noted that this value overestimates by far the actual contribution from (17) since the analysis of Ref. [18] is incapable of separating the contribution of ${ }_{\Lambda}^{4} \mathrm{H}+\pi^{+}$out of the global ${ }_{\Lambda}^{4} \mathrm{H}+\pi^{ \pm}$fraction. Note that a microscopic reaction evaluation of (17) requires a theoretical model which takes into account different channels ( $\Lambda$ production, $\Sigma$ production, compound nucleus formation) the weights of which are not experimentally known. We chose to avoid relying on a model and to assume, instead, an overly conservative evaluation of the background from the chain (17). The estimated level of this background is negligible with respect to the BGD1 background from the chain (16).

Other reaction chains have been considered, such as:

$$
\begin{aligned}
K_{\text {stop }}^{-}+{ }^{6} \mathrm{Li} \rightarrow & \Sigma^{+}+{ }_{\Lambda}^{3} \mathrm{H}+d+\pi^{-} \quad\left(p_{\pi^{-}} \leqslant 165 \mathrm{MeV} / c\right) \\
& \hookrightarrow n+\pi^{+} \quad\left(p_{\pi^{+}}<250 \mathrm{MeV} / c\right), \\
K_{\text {stop }}^{-}+{ }^{6} \mathrm{Li} \rightarrow & { }_{\Lambda}^{3} \mathrm{H}+3 n+\pi^{+} \quad\left(p_{\pi^{+}} \leqslant 242 \mathrm{MeV} / c\right) \\
& \hookrightarrow{ }^{3} \mathrm{He}+\pi^{-} \quad\left(p_{\pi^{-}} \sim 115 \mathrm{MeV} / c\right), \\
K_{\text {stop }}^{-}+{ }^{6} \mathrm{Li} \rightarrow & \Lambda+{ }^{3} \mathrm{H}+2 n+\pi^{+} \quad\left(p_{\pi^{+}} \leqslant 247 \mathrm{MeV} / c\right) \\
& \hookrightarrow p+\pi^{-} \quad\left(p_{\pi^{-}}<195 \mathrm{MeV} / c\right) .
\end{aligned}
$$

These reaction chains may be safely discarded since all of them involve too low values of $T\left(\pi^{+}\right)+T\left(\pi^{-}\right)$, less than $190 \mathrm{MeV}$, which are way outside the cut applied on $T_{\text {tot }}(\pi)$; the chain (19) may be discarded in addition by the cut imposed on $p_{\pi^{-}}$.

Finally, another mechanism which could produce a $\left(\pi^{+}, \pi^{-}\right)$pair in the final state is

$$
\begin{aligned}
K_{\text {stop }}^{-}+{ }^{6} \mathrm{Li} \rightarrow & { }_{\Lambda}^{6} \mathrm{He}+\pi^{0} \quad\left(p_{\pi^{0}} \sim 280 \mathrm{MeV} / c\right) \\
& \hookrightarrow{ }^{6} \mathrm{Li}+\pi^{-} \quad\left(p_{\pi^{-}} \sim 108 \mathrm{MeV} / c\right),
\end{aligned}
$$

followed by a reaction on another ${ }^{6} \mathrm{Li}$ nucleus:

$$
\pi^{0}+{ }^{6} \mathrm{Li} \rightarrow{ }^{6} \mathrm{He}+\pi^{+} \quad\left(p_{\pi^{+}} \sim 280 \mathrm{MeV} / c \text { in the forward direction }\right) .
$$

However, the kinematics of (21) rules out a contribution from this reaction chain when applying the cuts on $\pi^{-}$momenta. Moreover, the mean free path of a $\pi^{0}$ with momentum $\sim 280 \mathrm{MeV} / c$, $l_{\text {free }}<0.1 \mu \mathrm{m}$, strongly reduces the probability of the second reaction of the chain with respect to $\pi^{0}$ decay.

The main source of instrumental background could be the presence of fake tracks, due to fake signals from the detectors, misidentified as true events by the track reconstruction algorithms. For this purpose we analyzed the events with a $\pi^{+}$and a $\pi^{-}$emitted in coincidence with a $K^{-}$ stopping in a given nuclear target under the following criteria:

(i) events relative to target nuclei other than ${ }^{6} \mathrm{Li}\left({ }^{7} \mathrm{Li},{ }^{9} \mathrm{Be},{ }^{13} \mathrm{C},{ }^{16} \mathrm{O}\right)$ were selected with the same selection criteria $T\left(\pi^{+}\right)+T\left(\pi^{-}\right)=202-204 \mathrm{MeV}, p_{\pi^{+}}=250-255 \mathrm{MeV} / c$ and $p_{\pi^{-}}=130-137 \mathrm{MeV} / c$, as for ${ }^{6} \mathrm{Li}$. Incidentally, for events coming from the (16) reaction chain on nuclear targets heavier than ${ }^{6} \mathrm{Li}, T\left(\pi^{+}\right)+T\left(\pi^{-}\right)<202 \mathrm{MeV}$ by at least $2 \sigma_{T}$ so that this criterion actually selects the instrumental background exclusively. Only one event was found, coming from ${ }^{9} \mathrm{Be}$ target; 
(ii) events relative to the ${ }^{6} \mathrm{Li}$ targets were selected with values of $T\left(\pi^{+}\right)+T\left(\pi^{-}\right)=193-$ $199 \mathrm{MeV}$ so as to search for neutron-rich hypernuclei produced on the other targets. No events were found.

Taking into account the number of $K_{\text {stop }}^{-}$detected in ${ }^{6} \mathrm{Li}$ targets and in all the other targets, we concluded that $0.27 \pm 0.27$ fake events should be expected from ${ }^{6} \mathrm{Li}$ due to the instrumental background (BGD2).

Combining together the expected number of events arising from physical and instrumental backgrounds that affect our selected data, $0.16 \pm 0.07$ (BGD1) and $0.27 \pm 0.27$ (BGD2), a total of $0.43 \pm 0.28$ background events has been established. From this value, following Poisson statistics, we may state that the three observed ${ }_{\Lambda}^{6} \mathrm{H}$ candidate events do not belong to the background distribution with a confidence level of 99\%; the difference between the measured yield and the total expected background can thus be safely considered a ${ }_{\Lambda}^{6} \mathrm{H}$ signal. The probability of observing three or more events from the background fluctuation following Poisson distribution with $\mu=0.43$ (BGD1+BGD2) or $\mu=0.16$ (only BGD1) is 0.0096 or 0.0006 , respectively. In terms of a statistical significance $S$ defined by $S=C / \sqrt{\mathrm{BGD}}$, with a signal $C=3-\mathrm{BGD}$, the statistical significance of the signal is 3.9 or 7.1 , respectively.

\section{Production rate evaluation}

Using the background estimates of the last section, it is possible to evaluate the product $R$. $\operatorname{BR}\left(\pi^{-}\right)$, where $R$ is the ${ }_{\Lambda}^{6} \mathrm{H}$ production rate per stopped $K^{-}$and $\operatorname{BR}\left(\pi^{-}\right)$is the branching ratio for the two-body weak decay ${ }_{\Lambda}^{6} \mathrm{H} \rightarrow{ }^{6} \mathrm{He}+\pi^{-}$:

$$
R \cdot \operatorname{BR}\left(\pi^{-}\right)=\frac{3-\mathrm{BGD} 1-\mathrm{BGD} 2}{\epsilon\left(\pi^{+}\right) \epsilon\left(\pi^{-}\right) K_{\text {stop }}^{-}\left({ }^{6} \mathrm{Li}\right)}=(1.3 \pm 0.9) \cdot 10^{-6} / K_{\text {stop }}^{-} .
$$

In Eq. (23), $\epsilon\left(\pi^{+}\right)$and $\epsilon\left(\pi^{-}\right)$indicate the global efficiencies for $\pi^{+}$and $\pi^{-}$, respectively, including detection efficiency, geometrical and trigger acceptances and pattern recognition, reconstruction and selection efficiencies, all of which have been evaluated by means of the full FINUDA simulation code, well tested in calculations for other reactions in similar momentum ranges $[17,24,25] . K_{\text {stop }}^{-}\left({ }^{6} \mathrm{Li}\right)$ is the number of $K^{-}$detected at stop in ${ }^{6} \mathrm{Li}$ targets.

The value (23) has to be corrected for the purity of the ${ }^{6} \mathrm{Li}$ targets used, $90 \%$, for the 0.77 $\sigma_{T}$ cut applied to $T\left(\pi^{+}\right)+T\left(\pi^{-}\right)$, and for the fraction of ${ }_{\Lambda}^{6} \mathrm{H}$ decaying in flight. In Ref. [18] a contribution of $20 \%$ is reported for the decay in flight of ${ }_{\Lambda}^{4} \mathrm{H}$ produced on a ${ }^{7} \mathrm{Li}$ target; extending this value also to ${ }_{\Lambda}^{6} \mathrm{H}$ and considering that the cut applied to $p_{\pi^{-}}, 130-137 \mathrm{MeV} / c$, allows to accept about one half of the pions emitted in flight, a correction factor of $10 \%$ is evaluated. The corrected result is

$$
\left[R \cdot \operatorname{BR}\left(\pi^{-}\right)\right]_{\text {corr. }}=(2.9 \pm 2.0) \cdot 10^{-6} / K_{\text {stop }}^{-}
$$

By assuming $\mathrm{BR}\left(\pi^{-}\right)=49 \%$, in analogy to the weak decay ${ }_{\Lambda}^{4} \mathrm{H} \rightarrow{ }^{4} \mathrm{He}+\pi^{-}$[18], we find $R=(5.9 \pm 4.0) \cdot 10^{-6} / K_{\text {stop }}^{-}$, fully consistent with the upper limit (5) obtained previously by FINUDA [10]. Although no theoretical calculation of this capture rate has been reported to provide direct comparison, the order of magnitude of the rate determined here is compatible with the interval of values calculated for production of heavier neutron-rich hypernuclei [7] with stopped $K^{-}$mesons and, as expected, is approximately three orders of magnitude lower than the capture rate for the production of ordinary particle-stable $\Lambda$ hypernuclei. 


\section{Discussion}

The binding energy deduced from the three measured events listed in Table 1 was recorded in Eq. (14): $B_{\Lambda}\left({ }_{\Lambda}^{6} \mathrm{H}\right)=4.0 \pm 1.1 \mathrm{MeV}$ with respect to ${ }^{5} \mathrm{H}+\Lambda$, close to the value $B_{\Lambda}\left({ }_{\Lambda}^{6} \mathrm{He}\right)=$ $4.18 \pm 0.10 \mathrm{MeV}$ with respect to ${ }^{5} \mathrm{He}+\Lambda$ for the other known $A=6$ hypernucleus [2]. It is in good accord with the estimate $4.2 \mathrm{MeV}$ made originally by Dalitz and Levi Setti [1] and confirmed by Majling [3]. It is lower by $1.8 \mathrm{MeV}$ than the value $5.8 \mathrm{MeV}$ suggested by Akaishi et al. [13], leaving little room for an attractive contribution from a $\Lambda N N$ three-body force of a similar magnitude, $1.4 \mathrm{MeV}$, which in Akaishi's calculations arises from a coherent $\Lambda N-\Sigma N$ mixing model. This is consistent with a substantial weakening of $\Lambda N-\Sigma N$ mixing contributions for the excess $p$ shell neutrons in ${ }_{\Lambda}^{6} \mathrm{H}$ with respect to the strong effect calculated in the $s$-shell hypernucleus ${ }_{\Lambda}^{4} \mathrm{H}$ [15]. Indeed, recent shell-model calculations by Millener indicate that $\Lambda N-\Sigma N$ mixing contributions to $B_{\Lambda}$ and to doublet spin splittings in the $p$ shell are rather small, about $(10 \pm 5) \%$ of their contribution in ${ }_{\Lambda}^{4} \mathrm{H}$ [26]. Nevertheless, given the measurement uncertainty of $1.1 \mathrm{MeV}$, one may not conclude that this $\Lambda N N$ force contribution is negligible, but only that its influence appears considerably lower than predicted. For illustration, see Fig. 1.

It is possible to avoid considering explicitly the $\Lambda N-\Sigma N$ mixing effect in the evaluation of $B_{\Lambda}\left({ }_{\Lambda}^{6} \mathrm{H}\right)$ by updating the shell-model $(\mathrm{SM})$ argument used in Ref. [1]. We adopt a cluster model for ${ }_{\Lambda}^{6} \mathrm{H}$ in terms of ${ }_{\Lambda}^{4} \mathrm{H}$ plus two $p$-shell neutrons coupled to $J^{\pi}=0^{+}$as in ${ }^{6} \mathrm{He}$ g.s. The interaction of the $\Lambda$ hyperon with this dineutron cluster, including any $\Lambda \mathrm{nn}$ force arising from $\Lambda N-\Sigma N$ mixing, may be deduced from ${ }_{\Lambda}^{7} \mathrm{He}$ which consists of an $\alpha$ cluster plus precisely the same $\Lambda n n$ configuration under consideration in ${ }_{\Lambda}^{6} \mathrm{H}$. Subtracting $B_{\Lambda}\left({ }_{\Lambda}^{5} \mathrm{He}\right)=3.12 \pm 0.02 \mathrm{MeV}$ from $B_{\Lambda}\left({ }_{\Lambda}^{7} \mathrm{He}\right)$, with a value $B_{\Lambda}\left({ }_{\Lambda}^{7} \mathrm{He}\right)=5.36 \pm 0.09 \mathrm{MeV}$ obtained by extrapolating linearly from the known binding energies of the other members of the $A=7$ hypernuclear $T=1$ isotriplet (see Fig. 3, Ref. [27]), we obtain $2.24 \pm 0.09 \mathrm{MeV}$ for the $\Lambda n n$ sum of two-body and threebody interactions involving the $\Lambda$ hyperon. The value of $B_{\Lambda}\left({ }_{\Lambda}^{6} \mathrm{H}\right)$ is then obtained adding this $2.24 \mathrm{MeV}$ to $B_{\Lambda}\left({ }_{\Lambda}^{4} \mathrm{H}\right)=2.04 \pm 0.04 \mathrm{MeV}$ [2], so that $B_{\Lambda}^{\mathrm{SM}}\left({ }_{\Lambda}^{6} \mathrm{H}\right)=4.28 \pm 0.10 \mathrm{MeV}$. ${ }^{3}$ We have thus recovered the estimate originally made by Dalitz and Levi Setti [1].

As mentioned in the discussion of Table 1 , the ${ }_{\Lambda}^{6} \mathrm{H}$ mass values obtained from production are systematically higher than the corresponding values obtained from decay, leading to a mass difference of $\Delta M\left({ }_{\Lambda}^{6} \mathrm{H}\right)=0.98 \pm 0.74 \mathrm{MeV}$, see Eq. (15). This suggests that ${ }_{\Lambda}^{6} \mathrm{H}$ is produced in an excited state, while decaying from its ground state. We recall that Pauli spin is conserved in capture at rest. For $K_{\text {stop }}^{-}+{ }^{6} \mathrm{Li} \rightarrow{ }_{\Lambda}^{6} \mathrm{H}+\pi^{+}$production, since ${ }^{6} \mathrm{Li}$ is very well approximated (about $98 \%$ ) by a $L=0, S=1$ configuration [26], ${ }_{\Lambda}^{6} \mathrm{H}$ is dominantly produced in its $1^{+}$first excited state, decaying then by a fast magnetic dipole transition to the $0^{+}$ground state from which the mesonic weak decay occurs. In this situation, the pion kinetic energies $T\left(\pi^{+}\right)$and $T\left(\pi^{-}\right)$, directly measured by the FINUDA spectrometer, should reflect this systematic difference between production and decay. The mass of the $0^{+}$ground state should be calculated from the decay reaction only, giving a mean value $M\left({ }_{\Lambda}^{6} \mathrm{H}_{\mathrm{g} . \mathrm{s} .}\right)=(5800.9 \pm 1.2) \mathrm{MeV}$, corresponding to a binding energy of $4.5 \pm 1.2 \mathrm{MeV}$ with respect to $\left(\Lambda+{ }^{5} \mathrm{H}\right)$ and of $0.8 \pm 1.2 \mathrm{MeV}$ with respect to $\left({ }_{\Lambda}^{4} \mathrm{H}+2 n\right)$. For the $1^{+}$excited state it should be possible to evaluate a mean mass $M\left({ }_{\Lambda}^{6} \mathrm{H}^{*}\right)=$ $(5801.9 \pm 1.0) \mathrm{MeV}$. Although nominally unstable by $0.2 \pm 1.0 \mathrm{MeV}$, the low $Q$ value for two-

\footnotetext{
3 We thank Dr. D.J. Millener for alerting us to this estimate. A somewhat higher value, $B_{\Lambda}^{\mathrm{SM}}\left({ }_{\Lambda}^{6} \mathrm{H}\right)=4.60 \pm 0.24 \mathrm{MeV}$, is obtained if the preliminary value $B_{\Lambda}\left({ }_{\Lambda}^{7} \mathrm{He}\right)=5.68 \pm 0.03$ (stat) \pm 0.22 (syst) $\mathrm{MeV}$ from the $\left(e, e^{\prime} K^{+}\right)$reaction in the JLab E01-011 experiment is used [27].
} 
Table 2

Masses and $B_{\Lambda}$ values (in $\mathrm{MeV}$ ) of ${ }_{\Lambda}^{6} \mathrm{H}$ levels assuming that event 3 in Table 1 corresponds to the lowest two levels and events 1 and 2 correspond to higher levels.

\begin{tabular}{lllll}
\hline${ }_{\Lambda}^{6} \mathrm{H}$ & $0^{+}$ & $1^{+}$ & $2^{+}$ & $\left(1^{+}, 2^{+}, 3^{+}\right)$ \\
\hline$M$ & $5798.66 \pm 0.84$ & $5799.97 \pm 0.96$ & $5802.07 \pm 0.59$ & $5802.89 \pm 0.68$ \\
$B_{\Lambda}$ & $6.78 \pm 0.84$ & $5.47 \pm 0.96$ & $3.37 \pm 0.59$ & $2.55 \pm 0.68$ \\
\hline
\end{tabular}

neutron emission plus the associated $\Delta S=1$ spin flip required in the decay to ${ }_{\Lambda}^{4} \mathrm{H}\left(0_{\mathrm{g} . \mathrm{s} .}^{+}\right)+2 n$ are likely to make the ${ }_{\Lambda}^{6} \mathrm{H}\left(1_{\text {exc }}^{+} \rightarrow 0_{\text {g.s. }}^{+}\right) \mathrm{M} 1 \gamma$-ray transition competitive with the strong decay of the $1_{\text {exc }}^{+}$level.

It is worth noting that the uncertainty placed on the excitation energy $\Delta E\left(0^{+} \rightarrow 1^{+}\right)$, identifying this $\Delta E$ with $\Delta M\left({ }_{\Lambda}^{6} \mathrm{H}\right)$, is considerably smaller than the uncertainty of each one of the $0^{+}$and the $1^{+}$levels because $\Delta M$ has been determined directly from the sum of kinetic energies $T\left(\pi^{+}\right)+T\left(\pi^{-}\right)$and its associated uncertainty. The value determined in the present experiment for $\Delta E$ is smaller by $2 \sigma$ than the value $\Delta E_{\text {akaishi }}\left(0^{+} \rightarrow 1^{+}\right)=2.4 \mathrm{MeV}$ predicted in Ref. [13]. This is in line with the conclusion drawn from the absolute energy location of the $0^{+}$g.s., casting doubts on the applicability of the model developed by Akaishi et al. [13]. The value $\Delta E\left({ }_{\Lambda}^{6} \mathrm{H}\right.$ : $\left.0^{+} \rightarrow 1^{+}\right)=0.98 \pm 0.74 \mathrm{MeV}$ is in good accord with $\Delta E\left({ }_{\Lambda}^{4} \mathrm{H}: 0^{+} \rightarrow 1^{+}\right)=1.04 \pm 0.03 \mathrm{MeV}$ [2], consistently with a weak-coupling picture for the two 'halo' $p$-shell neutrons in ${ }_{\Lambda}^{6} \mathrm{H}$ outside the $s$-shell cluster of ${ }_{\Lambda}^{4} \mathrm{H}$.

It is also worth noting that the width of the selected $T\left(\pi^{+}\right)+T\left(\pi^{-}\right)$interval $(2 \mathrm{MeV})$ allows to include both production and decay pions within the experimental resolution of the mass determination at a $1 \sigma$ level, thus extending the validity of the working assumption on which the analysis method was based, namely that the masses of the produced and decaying hypernucleus are equal. A variation of the binding energy from the value of $5 \mathrm{MeV}$ used to fix the selection on $T\left(\pi^{+}\right)+T\left(\pi^{-}\right)$to $4 \mathrm{MeV}$, the average binding energy of the $0^{+}$and $1^{+}$levels, produces a completely negligible variation of the accepted fraction of events due to the selection criteria with respect to the errors. Finally, the absence of systematics arising from the particular choice of both width and position of the selected $T\left(\pi^{+}\right)+T\left(\pi^{-}\right)$interval indicates that the difference between the ${ }_{1}^{6} \mathrm{H}$ formation and decay masses is not influenced by the cut itself. It is important, however, to realize that the above deductions on the ${ }_{\Lambda}^{6} \mathrm{H}$ excitation spectrum rely on very scarce statistics and, therefore, have to be considered as indication, even if quite solid.

Before closing we wish to discuss briefly another scenario for the excitation spectrum of ${ }_{\Lambda}^{6} \mathrm{H}$ motivated by the somewhat large spread among the three ${ }_{\Lambda}^{6} \mathrm{H}$ candidate events. Apart from the $0^{+}$g.s. and $1^{+}$spin-flip excited state as in ${ }_{\Lambda}^{4} \mathrm{H}$, a $2^{+}$excited state as for the $p$-shell dineutron system in ${ }^{6} \mathrm{He}(1.80 \mathrm{MeV})$ is expected at about $2 \mathrm{MeV}$ excitation in ${ }_{\Lambda}^{6} \mathrm{H}$. Furthermore, a triplet of spin-flip excitations $1^{+}, 2^{+}, 3^{+}$, built on the $2^{+}$dineutron excitation is expected $1 \mathrm{MeV}$ higher, at about $3 \mathrm{MeV}$ excitation in ${ }_{\Lambda}^{6} \mathrm{H}$. It is then not unreasonable to assign event 3 in Table 1 to formation and decay of ${ }_{\Lambda}^{6} \mathrm{H}$ involving the $0^{+}$g.s. and its $1^{+}$spin-flip excited state, as considered above, whereas the other two events which are relatively close to each other correspond to formation of one of the $1^{+}, 2^{+}, 3^{+}$levels and to decay from the $2^{+}$dineutron excitation. This scenario generates an additional excitation scale to confront the $\approx 3 \mathrm{MeV}$ separation between the first two events of Table 1 and the third one. This results in the assignment of ${ }_{\Lambda}^{6} \mathrm{H}$ levels listed in Table 2.

The table exhibits that the g.s. of ${ }_{\Lambda}^{6} \mathrm{H}$ is bound in this scenario much stronger than the SM estimate outlined above, and in fact it is even more bound than predicted by Akaishi [13], al- 
though the excitation energy of the spin-flip $1^{+}$level appears considerably smaller than in his prediction. The excitation energy of the $2^{+}$level comes out about $3.4 \mathrm{MeV}$, too high with respect to the simple SM consideration. We conclude that this scenario is unlikely, but this conclusion does not derive from any model-independent experimental observation. Future experiments will tell.

\section{Conclusions}

We have reported the first observation of the hyper superheavy hydrogen ${ }_{\Lambda}^{6} \mathrm{H}$, based on detecting 3 candidate events that cannot be attributed to pure instrumental or physical backgrounds. The resulting binding energy of ${ }_{\Lambda}^{6} \mathrm{H}, B_{\Lambda}=4.0 \pm 1.1 \mathrm{MeV}$, agrees with simple shell-model estimates initiated by Dalitz and Levi Setti [1], but disagrees with the prediction made by Akaishi [13] based on a strongly attractive $\Lambda N N$ interaction within a coherent $\Lambda N-\Sigma N$ mixing model. It was suggested that the excitation energy of the $1^{+}$spin-flip state with respect to the $0^{+}$g.s. be identified with the systematic difference $\Delta M=0.98 \pm 0.74 \mathrm{MeV}$ between values of ${ }_{\Lambda}^{6} \mathrm{H}$ mass derived separately from production and from decay. This value is consistent with the $1.04 \mathrm{MeV}$ for the analogous spin-flip excitation in ${ }_{\Lambda}^{4} \mathrm{H}$, confirming again the applicability of the shell-model estimates. An experiment to produce ${ }_{\Lambda}^{6} \mathrm{H}$ via the $\left(\pi^{-}, K^{+}\right)$reaction on ${ }^{6} \mathrm{Li}$ at $1.2 \mathrm{GeV} / c$ was recently approved at J-PARC [28] and should run soon. The expected energy resolution is $2.5 \mathrm{MeV}$ FWHM, and the expected statistics about 1-2 orders of magnitude higher than previous KEK experiments.

\section{Acknowledgements}

We dedicate this article to the memory of our colleague Ambrogio Pantaleo, prematurely passed away. Dr. Pantaleo participated actively since the beginning to the FINUDA experiment and started the study of neutron-rich hypernuclei production in FINUDA, first results of which have been published in [10]; this article marks the completion of his dedicated contribution.

\section{References}

[1] R.H. Dalitz, R. Levi Setti, Nuovo Cimento 30 (1963) 489.

[2] M. Jurić, et al., Nucl. Phys. B 52 (1973) 1; Updated in: D.H. Davis, Nucl. Phys. A 754 (2005) 3c.

[3] L. Majling, Nucl. Phys. A 585 (1995) 211c.

[4] D.R. Tilley, et al., Nucl. Phys. A 745 (2004) 155.

[5] R.E. Chrien, C.B. Dover, A. Gal, Czech. J. Phys. 42 (1992) 1089.

[6] K. Kubota, et al., Nucl. Phys. A 602 (1996) 327.

[7] T.Yu. Tretyakova, D.E. Lanskoy, Nucl. Phys. A 691 (2001) 51c.

[8] P.K. Saha, et al., Phys. Rev. Lett. 94 (2005) 05202.

[9] T.Yu. Tretyakova, D.E. Lanskoy, Phys. At. Nucl. 66 (2003) 1681.

[10] M. Agnello, et al., Phys. Lett. B 640 (2006) 145.

[11] M. Agnello, et al., Phys. Rev. Lett. 108 (2012) 042501.

[12] A.A. Korsheninnikov, et al., Phys. Rev. Lett. 87 (2001) 092501.

[13] Y. Akaishi, T. Yamazaki, Frascati Physics Series XVI (1999) 59; K.S. Myint, T. Harada, S. Shinmura, Y. Akaishi, Few-Body Syst. Suppl. 12 (2000) 383;

K.S. Myint, Y. Akaishi, Prog. Theor. Phys. Suppl. 146 (2002) 599;

S. Shinmura, K.S. Myint, T. Harada, Y. Akaishi, J. Phys. G 28 (2002) L1;

Y. Akaishi, K.S. Myint, AIP Conf. Proc. 1011 (2008) 277;

Y. Akaishi, Prog. Theor. Phys. Suppl. 186 (2010) 378. 
[14] G. Audi, A.H. Wapstra, C. Thibault, Nucl. Phys. A 729 (2003) 337.

[15] Y. Akaishi, T. Harada, S. Shinmura, K.S. Myint, Phys. Rev. Lett. 84 (2000) 3539.

[16] M. Agnello, et al., Phys. Lett. B 685 (2010) 247.

[17] M. Agnello, et al., Phys. Lett. B 698 (2011) 219.

[18] H. Tamura, et al., Phys. Rev. C 40 (1989) R479.

[19] C. Vander Velde-Wilquet, et al., Nucl. Phys. A 241 (1975) 511.

[20] P.A. Katz, et al., Phys. Rev. D 1 (1970) 1267.

[21] H. Outa, Ph.D. thesis, University of Tokyo, 2003, unpublished.

[22] D.R. Tilley, et al., Nucl. Phys. A 708 (2002) 3.

[23] R. Barlow, C. Beeston, Comp. Phys. Comm. 77 (1993) 219.

[24] M. Agnello, et al., Nucl. Phys. A 775 (2006) 35.

[25] M. Agnello, et al., Phys. Lett. B 681 (2009) 139.

[26] D.J. Millener, Lect. Notes Phys. 724 (2007) 31.

[27] O. Hashimoto, et al., J. Phys. Conf. Ser. 312 (2011) 022015.

[28] A. Sakaguchi, et al., Production of neutron-rich $\Lambda$-hypernuclei with the double charge-exchange reaction, Proposal P10 (revised) for Nuclear and Particle Physics Experiments at J-PARC, http://j-parc.jp/NuclPart/Proposal_e.html. 